\title{
The Efficiency of Non-Contrast Computed Tomography in the Estimation of Urinary Stone Composition
}

\author{
Ilker Atici ${ }^{\mathrm{a}}$, Nuray Voyvoda ${ }^{\mathrm{b}, \mathrm{d}}$, Ozlem Tokgoz ${ }^{\mathrm{a}}$, Husnu Tokgoz ${ }^{\mathrm{c}}$
}

\begin{abstract}
Background: Prior knowledge of stone composition is key to determining stone brittleness as well as treatment management and prophylactic approach. The present study seeks to visualize stone type on non-contrast computed tomography on the basis of Hounsfield Unit (HU) values.
\end{abstract}

Methods: A retrospective evaluation was performed of non-contrast computed tomography scans of patients who underwent urinary system operation to remove stones which were subjected to biochemical analysis. The localization and size of the stones were determined and their HU values, mean attenuation/size (HUD) and maximum attenuation/size ratios were calculated.

Results: The results of stone analysis revealed 34 calcium phosphate, 11 calcium oxalate, 5 triple phosphate (struvite) stones. On the basis of measurement results, a significant difference was identified among HU values of the three stone types $(\mathrm{P}=0.002)$. When the stones were compared in pairs, this difference was established to be due to the difference between the densities of calcium phosphate and struvite stones. No significant difference was observed among the stone groups with regard to HUD and maximum attenuation/size ratios.

Manuscript accepted for publication October 27, 2011

aZonguldak Karaelmas University School of Medicine, Department of Radiology, Turkey

${ }^{\mathrm{b}}$ Kocaeli Acibadem Hospital, Department of Radiology, Turkey

'Zonguldak Karaelmas University School of Medicine, Department of Urology, Turkey

${ }^{\mathrm{d}}$ Corresponding author: Nuray Voyvoda, Kocaeli Acıbadem Hospital, Department of Radiology, Yeni Mah. Inkilap Cad. No:9, Derince,

Kocaeli, Turkey. Email: nuraykad@gmail.com

doi:10.4021/wjnu4e
Conclusions: HU values are a useful parameter to distinguish between calcium phosphate and struvite stones. The inclusion of HU values in reports will set the right course for treatment.

Keywords: Kidney stone; Computed tomography; Analysis

\section{Introduction}

The most significant recent advance in the imaging of renal calculi has been the development of non-contrast computed tomography (NCCT) and its use in the detection of ureteral and renal stones [1]. The speed, safety and accuracy of NCCT make it the method of choice in the assessment of patients with suspected urinary tract calculi [2-9].

Compared with plain radiography, ultrasound and intravenous urography, NCCT is superior in its ability to detect urinary calculi, to distinguish calculi from other abnormalities (e.g. blood clot, stricture or neoplasm) and to identify non-urological causes of flank pain. The ability of NCCT to assess stone size and location is well accepted, but less clear is its ability to determine stone composition [10].

Knowing the composition of a urinary calculus is frequently a key factor in determining its most appropriate management. The selection of the optimal treatment method (e.g. ureteroscopy, pyelolithotomy, percutaneous lithotripsy or extracorporeal shock wave lithotripsy) depends primarily on the chemical composition of the individual patient's calculus is essential. It is conceivable that the ability to predict stone composition before treatment would enable the urologist to group patients according to stone density and treat them accordingly. Thus, those patients with high density stones could be managed endoscopically rather than by ESWL, since stones composed of calcium oxalate monohydrate and cystine typically do not fragment well with ESWL [11].

While blood chemistries, examination of the urine sediment, and review of the patient's clinical history are helpful, the true nature of a calculus often remains in doubt [12]. To date, although several in vitro studies have investigated 
Table 1. A Comparison of the Stone Types With Regard to Age

\begin{tabular}{lllll}
\hline & CaP (n:34) & CaO (n:11) & Struvite (n:5) & P \\
\hline Age & $51.7 \pm 14.4$ & $42.6 \pm 12.5$ & $63.0 \pm 10.7$ & 0.024 \\
\hline
\end{tabular}

CaP: calcium phosphate; $\mathrm{CaO}$ : calcium oxalate.

whether NCCT could determine the chemical composition of urinary calculi, few in vivo studies have been performed. Hence, this study was undertaken to see if an in vivo Hounsfield Unit (HU) or HU density measurement could be used to predict the composition of urinary stones.

\section{Materials and Methods}

Of the patients who were admitted to our hospital between 2005 and 2010, those with suspected urinary tract stone disease were identified on the basis of prior stone history, physical examination and laboratory findings. The patients were included for study who were confirmed to have stones in the urinary system based on NCCT and whose stones were removed by operation and subjected to chemical analysis. Those patients with urinary tract catheters who were given contrast material intravenously or orally and underwent postoperative tomography were excluded from the study.

In addition, those cases were excluded from the study in which the number of cystine and calcium carbonate stones identified was not sufficient enough to achieve a significant overlap. The local ethics committee approved the study.

\section{CT protocol}

The CT scans were obtained by means of a Philips Secure Spiral CT scanner and a Toshiba Activion 16 CT scanner. The scans were performed by using a standard stone viewing protocol at $120-140 \mathrm{kV}, 79-260 \mathrm{~mA}$, a section in the $3-5$ $\mathrm{mm}$ range and a section thickness of $3-5 \mathrm{~mm}$.

\section{Image analysis}

The image analysis of each scan was carried out in two separate evaluating sessions by two staff radiologists (IA, NV) unaware of stone composition.

The radiologist who performed the assessment detected stones on the existing scans which were then removed by surgery. For each calculus, a region of interest was created overlying the whole calculus on the slice in which it was seen at its largest diameter.

The window width (WW) was adjusted to 2500 and the window level (WL) to 480 . The slice was magnified four fold, three different points were determined where the stone was observed to be the densest and the Hounsfield unit was measured of an area $1 \mathrm{~mm}$ on average. On the basis of these results, the mean $\mathrm{HU}$ value was recorded.

Hounsfield unit density (HUD) was also calculated for each stone by taking the mean HU for each stone and dividing it by the largest transverse diameter of the stone.

Next, the peak attenuation for the stones and the peak attenuation/size ratio were compared with the results of the stone analysis.

\section{Statistical analysis}

The statistical evaluation was conducted by means of SPSS (version 13.0). How well numerical variables fit a normal distribution was visualized using the Kolmogorov-Smirov test. The definitive statistics for numerical variables were expressed in average \pm standard deviation and those for categorical data in numbers and percentages. The differences between groups with regard to categorical variables and the relationships between variables were studied by means of Chi-square test. The assessment of the differences between the two measurements was undertaken by the paired t-test. One- way analysis of variance was employed in comparing the 3 stone types in terms of numerical variables that displayed normal distribution. A $95 \%$ confidence interval was obtained when evaluating the results. Results were considered significant when $\mathrm{P}<0.05$.

\section{Results}

The study population was a total of 50 patients, 39 men and 11 women ranging in age from 24 to 8 , whose average age was 50 .

The patients were admitted to our hospital with side pains or dysuria, 16 of the patients had one or more additional systemic diseases (14 had hypertension, 2 chronic renal insufficiency, 4 diabetes mellitus, 2 congestive cardiac insufficiency, 1 chronic obstructive pulmonary disease). One patient was receiving treatment for hypercalcemia.

The stone sizes ranged from minimum $5 \mathrm{~mm}$ to maximum $50 \mathrm{~mm}$ (mean $2 \mathrm{~cm}$ ), 22 of the stones were located in the right urinary system (15 in the kidney and 7 in the ureter) and 28 in the left urinary system (22 in the kidney and 6 in the ureter).

Stone analysis carried out revealed 34 calcium phosphate, 11 calcium oxalate and 5 struvite stones. 
Table 2. A Comparison of the Stone Types With Regard to Gender

\begin{tabular}{lllll}
\hline & $\begin{array}{l}\text { CaP } \\
\text { Number (\%) }\end{array}$ & $\begin{array}{l}\text { CaO } \\
\text { Number (\%) }\end{array}$ & $\begin{array}{l}\text { Struvite } \\
\text { Number (\%) }\end{array}$ & P \\
\hline Female & $7(20.6)$ & $2(18.2)$ & $2(40)$ & 0.620 \\
Male & $27(79.4)$ & $9(81.8)$ & $3(60)$ & \\
\hline
\end{tabular}

CaP: calcium phosphate; $\mathrm{CaO}$ : calcium oxalate.

A comparison of the stone types with regard to age identified a significant difference between them $(\mathrm{P}=0.024)$. This was found to be due to difference between the average age of the patients with struvite stones and that of the patients with calcium oxalate stones (Table 1).

No significant difference was noted when the stones were compared with regard to gender $(\mathrm{P}=0.620)$ (Table 2$)$.

The difference between the HU values of the 3 stone groups on the basis of, the measurements by the first and second observers was found to be significant $(\mathrm{P}=0.002$ and $\mathrm{P}=0.003$ ) (Table 3).

It was discovered that this difference was caused by the difference between the HU values for phosphate stones and those for struvite stones $(\mathrm{P}=0.003$ and $\mathrm{P}=0.004$, respectively). The difference among HU values for calcium phosphate-calcium oxalate and calcium oxalate-struvite stones was found not to be significant.

For two observers, when HUD was compared with regard to stone types no significant difference was observed among them $(\mathrm{P}=0.135$ and $\mathrm{P}=1.159)$ (Table3). When the ratio of the maximum stone density to stone size was compared with regard to stone type, no significance was observed among the stone types $(\mathrm{P}=0.137$ and $\mathrm{P}=0.194)$ (Table 3$)$.

The measurements conducted by the two observers were statistically significant $(\mathrm{P}=0.981)$.

Given a cut-off value which was assumed to be 80 for HUD, $8 \mathrm{CaP}, 2 \mathrm{CaO}$ and 1 struvite stones were found which had a HUD greater than 80 , and $26 \mathrm{CaP}, 9 \mathrm{CaO}$ and 4 struvite stones were identified whose HUD was less than 80 (Table 4).

\section{Discussion}

The choice of effective clinical management of urinary tract calculi can be facilitated by knowing the precise chemical composition of the stones and their corresponding fragility $[13,14]$.

Table 3. Values for HU, HUD and the Ratio of Maximum Stone Density to Stone Size Which Were Obtained by the Two Observers

\begin{tabular}{|c|c|c|c|c|}
\hline & $\mathrm{CaP}$ & $\mathrm{CaO}$ & Strüvit & $\mathbf{P}$ \\
\hline $\mathrm{HU}^{1}$ & $1082 \pm 403$ & $845 \pm 361$ & $444 \pm 194$ & 0.002 \\
\hline $\mathrm{HU}^{2}$ & $1079 \pm 417$ & $853 \pm 336$ & $448 \pm 188$ & 0.003 \\
\hline HUD $^{1}$ & $61 \pm 32$ & $43 \pm 26$ & $40 \pm 35$ & 0.135 \\
\hline HUD $^{2}$ & $61 \pm 33$ & $44 \pm 28$ & $39 \pm 33$ & 0.159 \\
\hline Max. HU/size ${ }^{1}$ & $66 \pm 32$ & $47 \pm 25$ & $44 \pm 38$ & 0.137 \\
\hline Max. HU/size ${ }^{2}$ & $68 \pm 35$ & $50 \pm 29$ & $45 \pm 35$ & 0.194 \\
\hline
\end{tabular}

$\mathrm{HU}^{1}$ : Hounsfield Unit value measured by first observer; $\mathrm{HU}^{2}$ : Hounsfield Unit value measured by second observer; HUD': Hounsfield Unit Density calculated by first observer; HUD²: Hounsfield Unit Density calculated by second observer; Max. HU/size ${ }^{1}$ : The ratio of maximum density to size calculated by first observer; Max. $\mathrm{HU} / \mathrm{size}^{2}$ : The ratio of maximum density to size calculated by second observer. 
Table 4. HUD Values Obtained by the Two Observers

\begin{tabular}{lccc}
\hline & CaP & CaO & Struvit \\
\hline HUD $>80$ & 8 & 2 & 1 \\
$\mathrm{HUD}<80$ & 26 & 9 & 4 \\
\hline
\end{tabular}

HUD: Hounsfield Unit Density; cutoff value: 80.

Indeed, predicting susceptibility to fragmentation in situ before treatment, notably extracorporeal shock wave lithotripsy and endoscopic laser lithotripsy, could be potentially useful [15]. Knowing the composition of these stones can also be useful for patients who are susceptible to dietary management or metabolic intervention [16].

Typically, pure stones composed of calcium oxalate monohydrate and brushite or cystine are relatively refractory to shock wave lithotripsy and percutaneous ultrasonic lithotripsy, and are more likely to be treated endoscopically whereas uric acid stones are usually treated with oral alkalinization $[13,14,17,18]$. Calcium oxalate dihydrate and struvite stones usually fragment easily with both shock wave lithotripsy and ultrasonic lithotripsy [16].

NCCT has emerged as the modality of choice in the evaluation of acute flank pain. The basic advantages of NCCT over other imaging methods include high sensitivity and specificity in the detection of ureteral and renal stones, speed, safety, detection of nonurological pathology and cost [19].

Contradictory findings have been published in the literature regarding the ability of helical CT to accurately assess the chemical composition of renal calculi [16]. These variations can be explained by the use of the different CT scanners, degree of collimation, energy setting, technique, stone size and perhaps interpretation of CT numbers [19].

The technique of CT scanning plays a role in measuring the HU values of urinary stones, specifically the size of collimation [20]. Saw et al. found that scanning stones that were smaller than the size of collimation subjected them to partial volume inaccuracies which had an impact on the measured HU values. They concluded that using a smaller collimation size permitted better accuracy in the prediction of stone composition [20]. Another parameter in the CT technique is dual energy scanning in which the differences in radiodensity observed by scanning stones at different energies are used for determination of their compositions [21, 22].

In our study, NCCT was performed with a collimation of 3 - $5 \mathrm{~mm}$ using single-energy scanning at $120-140 \mathrm{kV}$. The use of $1 \mathrm{~mm}$ collimation is clinically impractical in the routine evaluation of acute flank pain. Dual-kilovolt scanning also is not practical, and requires repeated imaging of the patient [23]. As we did not use stones less than $5 \mathrm{~mm}$ in our study, the partial volume effect due to collimation was minimalized.

The studies which Motley et al. carried out on 100 patients revealed that 87 patients had calcium stones, 7 uric acid stones, 4 struvite stones and 2 cystine stones. Their HU values were found to be $440 \pm 262,270 \pm 134,401 \pm 198$, $248 \pm 0$ respectively. They did not find a significant difference between the average HU values for these stone types [24].

Demirel et al., in their studies performed on 87 patients, discovered that 54 patients had calcium oxalate stones, 19 struvite stones and 14 uric acid stones, whose HU values were $812 \pm 135,614 \pm 121$ and $413 \pm 143$, respectively [19]. It was possible to distinguish between these three types of stones on the basis of their average HU values $(\mathrm{P}=0.001)$.

Patel et al. conducted an analysis of 100 stones and found that 56 of them contained calcium oxalate monohydrate $(\mathrm{CaOMH}), 16$ calcium oxalate dihydrate $(\mathrm{CaODH})$, 4 brushite, 9 apatite, 2 cystine and 13 uric acid. Based on their HU values, the cacium phosphate stones (brushite and apatite) are stones with the highest density just as our study demonstrated $(1123 \pm 254$ and $844 \pm 346)$. In the present study, $\mathrm{HU}$ values for $\mathrm{CaOMH}, \mathrm{CaODH}$, cystine and uric acid were established to be $879 \pm 230,517 \pm 205,550 \pm 74$ and $338 \pm 145$, respectively. The HU values of the calcium stones were found to display significant difference with regard to their subtypes [25].

Our studies revealed that calcium phosphate stones had a $\mathrm{HU}$ value of $1080 \pm 410$, calcium oxalate stones $849 \pm 348$ and struvite stones $446 \pm 191$. It was demonstrated that calcium phosphate and struvite stones can be distinguished on the basis of their HU values.

Motley et al. postulated that as stone size increases, so does the HU value and put forward the concept of Honsfield unit density (HU/stone size) to eliminate the effect of stone size. They found HUD values to be $105 \pm 43$ for calcium stones and $53 \pm 28$ for struvite stones, and reported that calcium stones could be distinguished from calcium-free ones [24]. However, our study clearly shows that HUD values do not make it possible to make a distinction among $\mathrm{CaP}, \mathrm{CaO}$ and struvite stones.

Nakada et al and Motley et al. argue that uric acid and $\mathrm{CaO}$ stones can be distinguished by assuming the cutoff val- 
ue to be $80[24,26]$. In contrast, a cutoff value of 80 assumed for our study failed to enable distinction between $\mathrm{CaO}, \mathrm{CaP}$, or struvite stones based on HUD.

During our study, when the ratio- of the maximum stone density to stone size was compared with regard to stone type, no significance was observed among the stone types.

In this and other in vivo studies, the densities of all the stone types were lower than those reported in in vitro studies $[20-22,24,26]$. As defined by Nakada et al., this is probably because of a volume-averaging effect from the surrounding soft tissues and the use of a smaller collimation size in in vitro studies [26].

In conclusion, NNCT in a patient who presents with acute flank pain provides information on stone size and localization as well as its content. Knowledge of hounsfield values of stones and their appearance in reports can determine the course of treatment.

The facts that calcium stones were not analyzed on the basis of their subtypes and not all stone types were identified during our study are our limitations. Larger clinical studies are required to assess this procedure further.

\section{References}

1. Older RA, Jenkins AD. Stone disease. Urol Clin North Am. 2000;27(2):215-229, vii.

2. Fielding JR, Steele G, Fox LA, Heller H, Loughlin KR. Spiral computerized tomography in the evaluation of acute flank pain: a replacement for excretory urography. J Urol. 1997;157(6):2071-2073.

3. Miller OF, Rineer SK, Reichard SR, Buckley RG, Donovan MS, Graham IR, Goff WB, et al. Prospective comparison of unenhanced spiral computed tomography and intravenous urogram in the evaluation of acute flank pain. Urology. 1998;52(6):982-987.

4. Fielding JR, Silverman SG, Rubin GD. Helical CT of the urinary tract. AJR Am J Roentgenol. 1999;172(5):11991206.

5. Dalrymple NC, Verga M, Anderson KR, Bove P, Covey AM, Rosenfield AT, Smith RC. The value of unenhanced helical computerized tomography in the management of acute flank pain. J Urol. 1998;159(3):735-740.

6. Vieweg J, Teh C, Freed K, Leder RA, Smith RH, Nelson RH, Preminger GM. Unenhanced helical computerized tomography for the evaluation of patients with acute flank pain. J Urol. 1998;160(3 Pt 1):679-684.

7. Sheley RC, Semonsen KG, Quinn SF. Helical CT in the evaluation of renal colic. Am J Emerg Med. 1999;17(3):279-282.

8. Boulay I, Holtz P, Foley WD, White B, Begun FP. Ureteral calculi: diagnostic efficacy of helical CT and implications for treatment of patients. AJR Am J Roentgenol. 1999; 172(6):1485-1490.
9. Katz DS, Hines J, Rausch DR, Perlmutter S, Sommer FG, Lumerman JH, Friedman RM, et al. Unenhanced helical CT for suspected renal colic. AJR Am J Roentgenol. 1999;173(2):425-430.

10. Kenney PJ. CT evaluation of urinary lithiasis. Radiol Clin North Am. 2003;41(5):979-999.

11. Dretler SP. Stone fragility--a new therapeutic distinction. J Urol. 1988;139(5):1124-1127.

12. Hillman BJ, Drach GW, Tracey P, Gaines JA. Computed tomographic analysis of renal calculi. AJR Am J Roentgenol. 1984;142(3):549-552.

13. Renner C, Rassweiler J. Treatment of renal stones by extracorporeal shock wave lithotripsy. Nephron. 1999;81 Suppl 1:71-81.

14. Dretler SP. Special article: calculus breakability--fragility and durility. J Endourol. 1994;8(1):1-3.

15. Logarakis NF, Jewett MA, Luymes J, Honey RJ. Variation in clinical outcome following shock wave lithotripsy. J Urol. 2000;163(3):721-725.

16. Bellin MF, Renard-Penna R, Conort P, Bissery A, Meric JB, Daudon M, Mallet A, et al. Helical CT evaluation of the chemical composition of urinary tract calculi with a discriminant analysis of CT-attenuation values and density. Eur Radiol. 2004;14(11):2134-2140.

17. Williams JC, Jr., Paterson RF, Kopecky KK, Lingeman JE, McAteer JA. High resolution detection of internal structure of renal calculi by helical computerized tomography. J Urol. 2002;167(1):322-326.

18. Rassweiler JJ, Renner C, Chaussy C, Thuroff S. Treatment of renal stones by extracorporeal shockwave lithotripsy: an update. Eur Urol. 2001;39(2):187-199.

19. Demirel A, Suma S. The efficacy of non-contrast helical computed tomography in the prediction of urinary stone composition in vivo. J Int Med Res. 2003;31(1):1-5.

20. Saw KC, McAteer JA, Monga AG, Chua GT, Lingeman JE, Williams JC, Jr. Helical CT of urinary calculi: effect of stone composition, stone size, and scan collimation. AJR Am J Roentgenol. 2000;175(2):329-332.

21. Mitcheson HD, Zamenhof RG, Bankoff MS, Prien EL. Determination of the chemical composition of urinary calculi by computerized tomography. J Urol. 1983;130(4):814-819.

22. Mostafavi MR, Ernst RD, Saltzman B. Accurate determination of chemical composition of urinary calculi by spiral computerized tomography. J Urol. 1998;159(3):673675.

23. Sheir KZ, Mansour O, Madbouly K, Elsobky E, AbdelKhalek M. Determination of the chemical composition of urinary calculi by noncontrast spiral computerized tomography. Urol Res. 2005;33(2):99-104.

24. Motley G, Dalrymple N, Keesling C, Fischer J, Harmon W. Hounsfield unit density in the determination of urinary stone composition. Urology. 2001;58(2):170-173.

25. Patel SR, Haleblian G, Zabbo A, Pareek G. Hounsfield 
units on computed tomography predict calcium stone subtype composition. Urol Int. 2009;83(2):175-180.

26. Nakada SY, Hoff DG, Attai S, Heisey D, Blankenbaker
D, Pozniak M. Determination of stone composition by noncontrast spiral computed tomography in the clinical setting. Urology. 2000;55(6):816-819. 